\section{P18 The Use of Guided Reading Questions as a Scaffolding Technique in a Flipped Graduate Metabolism Class}

Rachel L. Vollmer, PhD, RD, rvollmer@fsmail.bradley.edu, Bradley University, $1501 \mathrm{~W}$. Bradley Ave, Peoria, IL, 61625; Teresa Drake, PhD, CHES, RDN, Bradley University

Objective: Evaluate the use of guided reading questions (GRQ) as a scaffolding technique in a flipped classroom among graduate dietetic interns.

Use of Theory: The Staged Self-Directed Learning Model conceptualizes how students move towards self-directed learning in 4 stages. As students become more responsible for their own learning, they need different guidance or support from the teacher. In a previous study, students were initially resistant to flipped learning. It was hypothesized that if the instructor provided scaffolding techniques, such as GRQ, to help students learn on their own, the resistance to flipped learning may be minimized.

Target Audience: Graduate Dietetic Interns $(n=10)$ enrolled in a graduate-level metabolism course during their first semester at a midsize, private Midwestern university.

Course/Curriculum Description: In this flipped class, students read textbook chapters and/or watch videos prior to coming to class, then engage in activities (ie, case studies) to apply the information using team-based learning in class. GRQ were provided for students to use when reading the textbook for the first 5 weeks of the semester.

Evaluation Methods: Students completed 8 written learning reflections throughout the semester, which occurred approximately every other week. Content analysis was applied to these reflections to discover themes.

Results: Students viewed the GRQ as instructor support. Students reflected that they appreciated the GRQ at the beginning of the semester, but understood why they would not receive them for the entirety of the course. They reported that the GRQ helped them develop reading comprehension and note-taking skills. At the end of the semester, students reported that they would not need them.

Conclusions: Providing students GRQ in a flipped class, especially at the beginning of the semester, may be a useful scaffolding technique to help students gain skills in learning on their own and reading comprehension.

Funding: None.

\section{P19 Emotion- and Stress-Related Eating is Related to Weight Status Among College Students}

Caroline Brantley, MS, RD, cmbrantley@crimson.ua.edu, University of Alabama, 404 Russell Hall, Box 870311, Tuscaloosa, AL, 35475; Opeyemi Adewumi, BS, University of Alabama; Linda Knol, PhD, LDN, RDN, University of Alabama

Background: The Transactional Model of Stress and Coping proposes that stress occurs when one perceives that a particular stressor outweighs one's resources to cope. Emotion- and stress-related eating may be used as a measure to cope, which can lead to an increase in caloric intake and weight gain.

Objective: The purpose of this study was to understand the relationship between constructs of The Transactional Model of Stress and Coping and weight status among college students.

Study Design, Setting, Participants: Six hundred and ninety college students participated in an online survey.

Outcome Measure/Analysis: The survey included the Emotion- and Stress-related Eating and the Appraisal of Ability and Resources to Cope sub-scales from the Eating and Appraisal Due to Emotions and Stress (EADES) Questionnaire, the Perceived Stress Scale (PSS), self-report of height and weight, and demographic questions. Responses were captured on a 5 point Likert scale where lower scores represent greater emotion- and stress-related eating, lower appraisal of skills and resources to cope, and less stress. Logistic regression analysis was used to test the relationship between weight status and emotion- and stress-related eating, appraisal of resources to cope, and PSS while adjusting for demographics.

Results: In univariate analysis, when compared with healthy weight students, overweight/obese students had significantly lower Emotion-and Stress-related Eating scores $(3.3 \pm 0.7$ vs $3.7 \pm 0.8, P<.001)$ but similar PSS $(17.4 \pm 6.2$ vs $16.9 \pm 6.2, P=.23)$ and appraisal scores $(3.6 \pm 0.7$ vs $3.7 \pm 0.7, P=.10)$. After adjusting for demographics, overweight/obese individuals had significantly lower Emotion- and Stress-related Eating scores than their healthy weight counterparts regardless of perceived stress and appraisal to cope with stress (OR, 0.36; 95\% CI, 0.28$0.49)$.

Conclusions: Overweight and obese college students report more emotion- and stress-related eating compared to normal weight students; however, their perceived stress levels were similar. Emotion- and stress-related eating should be included in educational interventions.

Funding: None.

\section{P20 Effectiveness of Nutrition Interventions in Low-Income Rural and Urban Retail Environments: A Systematic Literature Review}

Linda Fergus, MS, RD, LDN, LFergus@agcenter.lsu.edu, Louisiana State University, 201 Knapp Hall, Baton Rouge, LA, 70803; Katherine Seals, MPH, LSU AgCenter; Denise Holston, PhD, RD, LDN, LSU AgCenter

Background: Retail interventions targeting food access and promoting healthy food choices are implemented to address the unique nutrition challenges of residents in low-income rural and urban environments. Research about the effectiveness of interventions in rural environments is limited.

Objective: The purpose of this systematic literature review is to identify successful retail interventions used in stores located in low-income rural and urban environments. 